\title{
Tangence
}

\section{Des faux amis aux frères ennemis : dialogue et conversation dans L’inquisitoire, Le square, L'amante anglaise, Le dîner en ville}

\section{From False Friends to Enemy Brothers: Dialogue and Conversation in L'inquisitoire (The Inquisitory), Le square (The Square),L'amante anglaise (The Lover), Le dîner en ville (The Dinner Party)}

\section{Marie-Hélène Boblet}

Numéro 79, automne 2005

Art et avatars de la conversation

URI : https://id.erudit.org/iderudit/012851ar

DOI : https://doi.org/10.7202/012851ar

Aller au sommaire du numéro

Éditeur(s)

Tangence

ISSN

1189-4563 (imprimé)

1710-0305 (numérique)

Découvrir la revue

Citer cet article

Boblet, M.-H. (2005). Des faux amis aux frères ennemis : dialogue et

conversation dans L'inquisitoire, Le square, L'amante anglaise, Le dîner en ville.

Tangence, (79), 55-73. https://doi.org/10.7202/012851ar

\section{Résumé de l'article}

Du dialogue à la conversation, de la conversation au dialogue : c'est de l'entretien de ces deux pratiques poétiques de l'échange verbal que traitera cet article. Après la Seconde Guerre mondiale, la mémoire du genre littéraire de la conversation se ranime, grâce au charme et à l'agrément de la vivacité, de la légèreté et de la fantaisie. Simultanément, les ambitions intellectuelles du dialogue, dont la pratique emprunte aux philosophes, séduisent des romanciers qui délaissent la narration de fiction et le romanesque pour le discours de l'enquête. Les nouveaux romanciers, en particulier, accusent la crise du roman et relancent le genre sur de nouvelles voies : quête de mémoire, de savoir, dialogue entre les âmes, communication intérieure combinent la vocation heuristique du dialogue et la séduction quasi érotique de la conversation. 


\title{
Des faux amis aux frères ennemis : dialogue et conversation dans L'inquisitoire, Le square, L'amante anglaise, Le diner en ville \\ Marie-Hélène Boblet, Université Paris III-Sorbonne nouvelle
}

\begin{abstract}
Du dialogue à la conversation, de la conversation au dialogue: c'est de l'entretien de ces deux pratiques poétiques de l'échange verbal que traitera cet article. Après la Seconde Guerre mondiale, la mémoire du genre littéraire de la conversation se ranime, grâce au charme et à l'agrément de la vivacité, de la légèreté et de la fantaisie. Simultanément, les ambitions intellectuelles du dialogue, dont la pratique emprunte aux philosophes, séduisent des romanciers qui délaissent la narration de fiction et le romanesque pour le discours de l'enquête. Les nouveaux romanciers, en particulier, accusent la crise du roman et relancent le genre sur de nouvelles voies: quête de mémoire, de savoir, dialogue entre les âmes, communication intérieure combinent la vocation heuristique du dialogue et la séduction quasi érotique de la conversation.
\end{abstract}

À la faveur de la crise du genre narratif et de l'imaginaire romanesque qui caractérise l'après-guerre, le discours se déploie dans tous ses atours et occupe le territoire laissé vacant par le récit. La «cure d'amaigrissement du roman» qui définit, selon certains, l'aventure du Nouveau Roman est, on le sait, favorable d'une part à l'expansion de la description jusqu'à l'ekphrasis et propice d'autre part aux échanges entre personnages. Car les personnages sont devenus parlants plutôt qu'actants, bavards plus que héros. Les drames s'assimilent à des drames de paroles, les actes se redéfinissent comme actes de paroles, c'est-à-dire comme autant d'actions qui agissent sur le réel en transformant, à l'intérieur du monde fictionnel, bien sûr, les relations humaines par le seul fait d'échanges verbaux et de relations discursives attribués aux 
personnages et distribués entre eux. Le déficit dont souffrent l'Histoire et l'imaginaire épique qui s'y accorde, le soupçon qui pèse sur la psychologie traditionnelle des personnages (qu'il s'agisse de la modalité balzacienne selon Robbe-Grillet ou de la modalité françois-mauriacienne selon Sartre) favorisent la substitution du parleur au personnage de roman, comme l'indiquent les titres de Louis-René des Forêts (Le bavard, 1947) ou de Nathalie Sarraute (Vous les entendez?, 1972 ; Disent les imbéciles, 1976).

Cette conversion ne concerne pas seulement les thèmes, mais affecte aussi le roman lui-même comme genre. Sur le plan thématique, la temporalité est bouleversée, la réponse à la question: de quoi s'agit-il? ne supposant plus un drame qui aura lieu dans le devenir et auquel serait suspendue la dynamique narrative, mais une action qui a déjà eu lieu et dont on parle. Premier exemple: dans L'inquisitoire (1962) de Pinget, un vieux domestique sourd est interrogé par deux inconnus, jamais identifiables autrement que par leur rôle discursif, qui cherchent à lui extorquer, sur un mode inquisitorial, des choses peu avouables sur son patron, sans doute peu recommandable. Mais si celui-ci s'est enfui et qu'on l'a perdu de vue, il est néanmoins défendu par son ancien serviteur. Un drame a dû avoir lieu dont nous ne saurons rien, sinon qu'il suscite curiosité et convoitise, volonté de savoir et d'obtenir des aveux chez des interrogateurs qu'on dirait tout droit sortis du Procès de Kafka. Autre exemple plus tardif: Tu ne t'aimes pas (1989), de Sarraute, reconstitue une conversation intime entre les différents moi d'un je qui s'est, dans une situation mondaine, exposé jusqu'à s'entendre répliquer "Vous ne vous aimez pas ». Les moi, affolés par un tel diagnostic, tentent de revivre la scène du drame et de s'expliquer comment un des leurs s'est verbalement comporté pour s'attirer et mériter cet arrêt. Ces romans - qu'il faut bien baptiser ainsi puisque leurs auteurs, qui sont aussi dramaturges, ont fait entrer ces œuvres dans cette catégorie générique - ont évacué l'arsenal romanesque, expulsé narration et commentaires. Ils se constituent exclusivement de dialogues apparentés à une procédure judiciaire: les métaphores de l'inquisitoire chez Pinget (terme médiéval désignant l'interrogatoire, puis le répertoire), du déambulatoire ou du parloir chez Sarraute explicitent un imaginaire commun à ces deux avocats devenus écrivains. "L'inquisition est le principe de l'interrogatoire cruel et systématique que j'ai adopté pour mon livre L'inquisitoire. J'ai forgé le substantif d'après un adjectif juridique ancien, la 
procédure inquisitoire ${ }^{1} »$. Le scénario implicite du tribunal suggéré par le titre de Pinget parle aux metteurs en scène, d'autant plus qu'il nourrit d'autres essais: L'hypothèse, en 1961, annonce déjà L'inquisitoire, dont se fait l'écho, en 1965, Autour de Mortin. L'affaire Ducreux (1995) confirme ce script quasi obsessionnel: on demande à un serviteur s'il connaissait le petit Ducreux, neveu orphelin adopté puis abandonné par Monsieur: le «on» renvoie à un diktat intérieur, puis finalement à une voix off. Autant de voix qui enquêtent, questionnent, spéculent, conjecturent. Du côté de Nathalie Sarraute, Tu ne t'aimes pas fait actuellement l'objet d'une adaptation scénique de Grégoire Ingold, interpellé par la situation de l'assemblée délibérative des moi. De même, le roman de Sarraute fait, pour sa part, réentendre les voix de pièces de théâtre: Pour un oui ou pour un non (1982) aborde la question du bonheur ou de la réussite, Isma (1970) ou C'est beau (1973) celle du jugement de valeur social ou esthétique.

La grande cohérence de chacune de ces œuvres saute aux yeux. En premier lieu, le déplacement chronologique du drame (drame que ne déroule plus le récit du roman, puisqu'il est antérieur à la fiction du texte qui s'appuie sur lui) impose le temps de la présence, celui du discours en acte, l'instant de l'énonciation. Les temps du passé sont réservés aux segments narratifs insérés dans le continuum discursif qui dicte l'organisation de la temporalité. L'effet de présence, la présentification de la fiction actualise l'imaginaire, qui s'impose ici et maintenant dans l'expérience de la lecture. En second lieu s'observe une hybridation générique, à laquelle étaient destinés, pour ainsi dire, ces écrivains de la voix, du ton, du Verbe en un mot. Le dialogue, au sens dramatique, y occupe tout l'espace textuel; les mots prennent la parole et sont les acteurs, comme dans le dernier texte de Nathalie Sarraute, Ouvrez (1997) : «Des mots, des êtres vivants parfaitement autonomes, sont les protagonistes de chacun de ces drames ${ }^{2}$.» L'entretien se réclame aussi d'une valeur heuristique traditionnellement associée au dialogue philosophique: c'est à la recherche de la vérité que s'acharnent les moi et les interrogateurs, et que coopèrent le moi verbeux qui s'est pris pour je et le vieux domestique sourd. Ces deux écrivains reprennent à nouveaux frais la psychologie, sans le réalisme explicatif ni la convention de l'intériorité transparente des

1. Robert Pinget et Madeleine Renouard, Robert Pinget à la lettre. Entretiens avec Madeleine Renouard, Paris, Belfond, 1993, p. 96.

2. Nathalie Sarraute, Ouvrez, Paris, Gallimard, 1997, p. 9. 
personnages. Ils observent et scrutent un tréfonds (Pinget), des tropismes (Sarraute), et l'enchevêtrement du dit et du non-dit dans la communication interpersonnelle. Il y va d'un nouveau réalisme, proprement phénoménologique, qui enregistre des manifestations langagières, les laisse signifier et se ramifier.

Dans ces mêmes années, d'autres auteurs exploitent de nouveaux territoires romanesques: Claude Mauriac se livre à une expérience d' "alittérature», dans un esprit d'exploration poétique et esthétique proche du laboratoire de Michel Butor. Le dialogue intérieur est un opus de quatre romans dont le second et le troisième sont intégralement discursifs, et qui regroupe Toutes les femmes sont fatales (1957), Le dîner en ville (1959), La marquise sortit à cinq heures (1961) et L'agrandissement (1963). Parallèlement, Marguerite Duras écrit, en 1955, Le square, dont une version abrégée est montée dès 1956 par Claude Martin, puis une version définitive en 1965 par Alexandre Astruc, au Théâtre Daniel Sorano $^{3}$. En 1958, elle écrit Les viaducs de la Seine-et-Oise, monté en 1959 à Marseille, puis en 1963 par Claude Régy, dont Beckett admira la mise en scène. Elle reniera pourtant cette pièce et, en 1967, composera à partir d'elle L'amante anglaise, roman dialogué, qui sera vite suivi du Théâtre de l'amante anglaise (1968). Le glissement du roman au théâtre laisse intact l'essentiel: le respect de la parole vive, le drame de l'échange, la célébration éthique du dialogue. Pour en finir avec cette question des genres, qui n'est pas le cœur de notre propos, rappelons l'extrême imbrication des versions. Le retour sur texte, les variations sur le même mettent en lumière une obsession, celle d'un imaginaire de la parole et de l'échange dont nul ne peut sans mutilation faire le deuil. Pour Duras comme pour Beckett, la pertinence du concept de genre est mise en débat et en doute; il n'y a pas de cloisons, mais une œuvre qui s'impose comme recherche d'une unité esthétique, synthétique et donc transgénérique. La totalité du grand ouvre dépasse les inscriptions locales de ses avatars. Les modalités lyrique, épique et dramatique s'entrelacent dans une symphonie postromantique, qui rendent hommage à l'intuition de Schelling selon lequel le drame est le plus synthétique des genres, car il fait parler les personnages et représente le monde. Les «romans-drames» de Pinget, Duras ou Claude Mauriac exemplifient cette ambition poétique,

3. En 1965 parait le premier tome du théâtre de Marguerite Duras aux éditions Gallimard. 
dans la mesure même où ce sont des romans dénarrativisés et dialogués.

Nous nous proposons de suivre les aventures d'écriture dans lesquelles s'engage chacun de ces écrivains à la lumière de l'opposition rhétorique entre dialogue et conversation. Le dialogue est-il le faux ami, voire le frère ennemi de la conversation? La présomption intellectuelle et opératoire de l'un contredit-elle la fantaisie fortuite et la vivacité mondaine de l'autre? De la même façon que roman et drame fusionnent à un moment précis du $\mathrm{Xx}^{\mathrm{e}}$ siècle, le dialogue et la conversation ne s'épousent-ils pas pour obtenir mieux et plus de la parole partagée? D'un côté, certes, le dialogue heuristique, fût-il inquisitoire, se rappelle ses origines socratiques, ses accointances avec l'ordre du vrai ou du vérifiable, avec la pratique du logos. Mais les réponses hyperinformatives du domestique de L'inquisitoire et les coqs-à-1'âne auxquels il se livre font éclater le cadre de l'interrogatoire et laissent miroiter ce qui pourrait être une conversation entre honnêtes gens, dont il a la nostalgie. Dans Le square, la conversation permet aux parleurs de fabriquer de la pensée à partir de leurs paroles, sans qu'ils y songent ou le recherchent rageusement. Si la maïeutique opère, c'est par la grâce désintéressée d'un bavardage, et la conversation dévie vers un dialogue aux enjeux philosophiques, confrontant deux types de sagesse. Enfin, dans Le dîner en ville, la conversation de salon se trouve contaminée par la communication entre les âmes, le «dialogue intérieur». Des glissements symétriques se repèrent, un dialogue peut en cacher un autre; en tout cas, dans la littérature romanesque postnarrative des années 1950 à 1970, le Verbe est célébré au nom de la Vie plus que du discours. La conversation fait dévier, vibrer, dériver le dialogue, le désordonne et le désaxe, là où le dialogue infiltre du sérieux philosophique, voire métaphysique, dans un échange conversationnel rituel et bourgeois. Quand la parole "travaille», c'est-à-dire lorsqu'elle donne aux entretiens une dimension épistémologique, parallèlement, la parole "littéraire», construite comme objet poétique de discours, investit la frontière entre les genres. C'est donc à ces deux imaginaires de l'échange et à leur rencontre que nous consacrons cet article, à la lumière des exemples privilégiés que sont L'inquisitoire, Le square, L'amante anglaise et Le dîner en ville. 


\section{L'inquisitoire (1962)}

«C'est l'oralité qui m'intéresse, j'écris pour l'oreille [...]», "J'entends ce que j'écris [...]", dit Robert Pinget: "Chez moi, le personnage ne se maintient que par la voix ${ }^{4} »$. Voix et oralité sont les deux sources d'inspiration de l'écrivain, l'ossature et la chair des personnages. L'injonction de répondre marque le début de L'inquisitoire, qui mime ironiquement l'injonction de Jérôme Lindon lancée à Robert Pinget de composer son roman pour les Éditions de Minuit: "Oui ou non répondez ${ }^{5}$ ». Ce premier échange entre interrogateurs et interrogé a été suivi de 500 pages, car l'humour a eu raison de la rétention - l'humour, mais aussi une interrogation sur le pouvoir de la parole et, plus particulièrement, de la question, avec ce qu'elle induit, entraîne, suppose d'implicite. Ce roman expose la rouerie et montre comment s'en défendre. Il donne le mot de la fin au non-dit, à tout «ce que vous oubliez de me demander ", à tout ce qu'on ne voit pas parce que ça nous crève les yeux: "[...] je fais l'effort je le fais même trop et le vrai se trouve à côté, ce que je ne sais plus ou que je ne sais pas encore ou que vous oubliez de me demander [...] je ne sais que des ragots je ne sais pas la vérité [...] la vérité c'est d'avoir sous les yeux quelque chose qu'on ne voit pas parce qu'on a autre chose à faire $[\ldots] »(I$, p. 317-321). Dans la mesure même où il reconstruit sémiotiquement un interrogatoire de type judiciaire, le roman exhibe les compétences rhétoriques des partenaires fictifs de ce jeu du chat et de la souris, et il sollicite celles des lecteurs qui coopèrent au jeu du roman. Les contraintes propres aux règles du discours qui président aux échanges sont exacerbées dans un contexte de traque de la vérité, voire de la faute, et toute dérogation à la règle est signifiante, stratégique. Si l'on se fonde sur les "lois du discours» repérées par Ducrot ou sur les «maximes conversationnelles » que Grice a élaborées ${ }^{6}$, on relève, sous l'égide

4. «Voix», entretien de Michel Crépu avec Robert Pinget, Esprit, Paris, février 1983, p. 11-12.

5. Il s'agit de la toute première phrase de L'inquisitoire, qui lance le roman; voir Robert Pinget, L'inquisitoire, Paris, Union générale d'éditions, coll. "10/18», 1971. Désormais, les références à cet ouvrage seront indiquées par le sigle $I$, suivi de la page, et placées entre parenthèses dans le corps du texte.

6. Voir Oswald Ducrot, "Les lois du discours", Langue française, Paris, $n^{\circ} 42$, mai 1979, p. 24-29, H. Paul Grice, "Logique et conversation», Communications, Paris, no 30, 1979, p. 57-72, et Jacques Moeschler, "Théorie pragmatique, acte de langage et conversation. Réaction à l'article d'A. Trognon et C. Brassac ", Cahiers de linguistique française, Genève, n ${ }^{\circ}$ 13, 1992, p. 108-124. 
de la règle de coopération, les lois de pertinence (maxime de relation), d'informativité (maxime de quantité, voire d'exhaustivité), de clarté (maxime de modalité) et de sincérité (maxime de qualité). Le silence menace toute communication, le mensonge et la rétention d'informations toute prétention au vrai, les coqsà-1'âne toute directivité et tout axe. La liberté et la fantaisie sont pourtant à ce prix. Le destinataire ne se contente pas d'un rôle passif d'auditeur répondant à des assignations, il interprète les intentions et les actes de communication qui lui sont adressés. Cette activité herméneutique lui assigne une responsabilité, certes secondaire et non initiale, mais qui lui permet de relancer le dialogue, de le diriger ailleurs et de l'orienter finalement. L'interprétation repose sur un calcul logique, inférentiel, basé sur des composantes rhétoriques et situationnelles plus que linguistiques. La compréhension des contenus littéraux soumis à des règles sémantiques stables doit se doubler de la reconnaissance et de l'actualisation de contenus dérivés, sous-entendus, allusifs, qui se trouvent souvent au cœur de l'enjeu de l'échange langagier. Le dit, le message communiqué reçu est entendu s'il s'assortit d'une anticipation des conséquences possibles de l'énoncé sur le plan de l'échange et de la situation énonciative. Ainsi le domestique, doté d'une compétence rhétorique et interprétative qui s'aiguise au fil de l'entretien, comprend qu'on veut «lui tirer les vers du nez» ( $I$, p. 473): «J'en ai assez de votre système vous essayez de me faire tromper sur des détails vous feriez mieux de me demander les choses importantes» (I, p. 411). Mener une pareille joute verbale exige de lui qu'il impose son thème et digresse librement, comme s'il conversait, alors qu'on l'interroge. L'analogie et le décousu caractérisent ses répliques, lesquelles témoignent du plaisir de parler et du désir de reconquête de son autonomie. Ses détournements discursifs lui valent des rappels à l'ordre l'invitant à mieux respecter les règles qui doivent régir le dialogue engagé ici: celles de sincérité et d'informativité ( Comment peut-on vous croire lorsque plusieurs fois déjà vous avez essayé de dissimuler la vérité?» $[I$, p. 374]); et celles de pertinence et d'exhaustivité («Il est difficile de vous croire après vos louvoiements et omissions continuels» $[I$, p. 473]). Or, finalement, c'est l'accusé qui exprimera une définition nouvelle et stricte de la responsabilité rhétorique et dénoncera l'artifice de l'argumentation judiciaire au nom de la logique du vivant:

[...] si je les avais dites posément en prenant mon temps elles n'auraient pas été pareilles au lieu que comme ça vous allez en 
tirer avantage sur ces messieurs ou sur les autres ou sur moi [...] avant quand on n'a pas l'expérience on est comme ça toujours pressé de se faire des idées avec des bribes et tous nos jugements sont faux par le fait [...] il n'y a de conséquences que celles qu'on imagine sur le moment ou qu'on force à s'appeler conséquences, les vraies ne leur ressemblent pas du tout elles se combinent avec tout le reste et c'est la vie [...] $(I$, p. 473).

Le soupçon qui pèse sur le logos et ses prétentions achèvera de discréditer toute pratique de l'interrogation fondée sur la prérogative, sur une distribution des places et un rapport de pouvoir iniques et instrumentalisés pour faire faire, faire dire et faire avouer quelque chose à quelqu'un. La pratique de l'aveu est dénoncée par Pinget comme, à la même époque, par Michel Foucault:

C'est un rituel qui se déploie dans un rapport de pouvoir, car on n'avoue pas sans la présence au moins virtuelle d'un partenaire qui n'est pas simplement l'interlocuteur, mais l'instance qui requiert l'aveu, l'impose, l'apprécie, et intervient pour juger, punir, pardonner, consoler, réconcilier; un rituel où la vérité s'authentifie de l'obstacle et des résistances qu'elle a eu à lever pour se formuler ${ }^{7}$.

Questionner est non seulement une pratique mais un savoirfaire, un art dont le domestique devient le défenseur. Il ne convient pas d'interroger n'importe qui, car le questionné doit lui-même offrir des dispositions physiques et mentales dont le domestique est dépourvu («je ne suis pas curieux de nature [...] je suis sourd comme un pot $[\ldots] »[I$, p. 8$]$; " vous oubliez que je suis sourd $[\ldots] »[I$, p. 103]). Surtout, l'interrogateur doit savoir répondre de la légitimité de ses sous-entendus et de la justesse des présupposés de la requête. À défaut, l'interrogé résistera aux interrogateurs: «Veuillez préciser une fois pour toutes le genre d'histoires. - Estce qu'il y a un genre d'histoires il y en a mille quand on ne s'entend pas avec les gens» ( $I$, p. 136). Un sous-entendu est décelé dans le moindre mot des interrogateurs: "Qu'entendez-vous par cherchent les influences qui leur plaisent. - Rien d'autre que ce que j'entends» $(I$, p. 92); «Je me demande bien où vous voulez en venir, comment voulez-vous que je sache» $(I$, p. 71). Jusqu'à la déflagration finale:

Non je ne répondrai pas je ne veux pas répondre madame Lemove n'est rien il n'y a rien à en dire et de me forcer à en

7. Michel Foucault, Histoire de la sexualité, Paris, Gallimard, 1976, t. I, p. 82-83. 
parler n'ajoutera rien, c'est à moi de vous demander qu'est-ce que vous voulez savoir qu'est-ce que vous voulez me tirer, je ne sais que des ragots je ne sais pas la vérité jamais et nous en crèverons de ne pas savoir et tous ceux qui vous ressemblent ils s'acharnent à découvrir le secret des autres ils crèveront avant eux ils seront à bout, ils ne savent pas demander ils savent préparer des réponses ce n'est pas comme ça qu'on demande [nous soulignons] (I, p. 319).

La manière de questionner trahit la (dé)loyauté de l'interrogateur, dicte la justesse de la réponse et ouvre un passage à la vérité, comme par défaut, en creux, de biais. Une remarque comme «ce n'est pas comme ça qu'on demande» $(I$, p. 319) rappelle l'exigence socratique de disponibilité, nécessaire au travail de l'esprit. Préparer des réponses revient à poser des questions rhétoriques, tandis que laisser ouverts les possibles fonde une éthique de l'entretien. Dans l'Antiquité, la pratique philosophique du dialogue croise l'exigence critique de type scientifique (que réélaborèrent le cartésianisme puis l'Auflklärung) avec le respect éthique de l'autre et de l'altérité, fût-elle irréductible au logos, à la connaissance. C'est le procès d'une certaine déviation de la pensée occidentale qu'ouvre Pinget, au nom d'une autre relation à la différence qu'il ne s'agit pas de réduire au même, mais de maintenir en son écart pour sauver la proximité plutôt que l'identité. L'inquisitoire met à la question l'art moderne de la réflexion, art logique et rhétorique désormais à bout de souffle. Par l'inachèvement, la non-totalisation de la fiction et de sa mise en discours, et par le jeûne auquel est soumis le diligent lecteur, le roman offre donc un nouveau modèle pour penser le rapport au vrai et la relation à autrui : aucun savoir n'échappe à la subjectivité, à la fragmentation, à la relativité; aucun sujet n'échappe au devoir d'écoute, à la patience d'accoucher d'un «fatras de bribes» qui seront «le reste» dont devra se satisfaire notre appétence encyclopédique.

À l'époque bénie du socratisme, la Raison est à la fois le Destinateur et le Destinataire de la quête qu'entreprend le dialogue, elle la commande et l'évalue. Mais cheminer vers la connaissance de l'idée, après la Seconde Guerre mondiale et l'Holocauste, réclame des sentiers de traverse qui court-circuitent les impasses de la modernité rationaliste, ses apories et surtout ses embuscades. Après tout peut-être s'agit-il, plus que d'une crise symptomatique de la postmodernité, d'un retour à l'Ancien, d'une Renaissance humble et patiente, après la déviation autoritaire du 
socratisme par le platonisme et la démesure orgueilleuse des fastes des Lumières? Selon Francis Jacques, "[1] e platonisme est le moment où le processus de questionnement s'autonomise sous la prérogative du maître. Le platonisme a transformé le dialogue de Socrate en une sorte de monologue ${ }^{8}$. " Victor Schmidt rend hommage à l'inventivité non dogmatique du dialogue socratique, de la méthode qui cherche et se cherche ${ }^{9}$. Lacan, de son côté, souligne la puissance épiphanique du dialogue, le surgissement révolutionnaire d'un savoir insu et d'une connaissance aveugle. C'est à cette épreuve de l'entretien, de «l'entre deux sujets» qu'on doit l'apparition de la question qui convient et de la réponse juste. "Ce que Socrate appelle, lui, l'episteme, la science, ce qu'il découvre en somme, ce qu'il dégage, ce qu'il détache, c'est que le discours engendre la dimension de la vérité ${ }^{10}$. " Il s'agit d'attraper du vrai au lancer d'une question, du vrai fait d'expérience subjective. À cet égard, les dialogues durassiens se composent aussi de fragments de souvenirs métissés de désirs, d'éclairs de clairvoyance, de suppositions qui emboîtent dans le roman dialogué une fiction au second degré : le roman dans la tête des personnages. Chercher une vérité sur la vie ou sur un crime, telle est l'ambition des deux romans dialogués qui célèbrent la parole et son pouvoir divinatoire, révélateur, et où l'on rejoint Socrate audelà de Platon: "Aporia, par définition et par structure, n'a rien à donner que son manque approximatif (aporia) constitutif. "Donner ce qu'on n'a pas" se trouve en toutes lettres à l'indice 202a du texte du Banquet. C'est exactement la formule calquée à propos du discours. Il s'agit de donner un discours, une explication valable, sans l'avoir» ( $T$, p. 148). Non pas savoir et dire qu'on ne sait rien, mais dire ce qu'on ne sait pas savoir ni avoir à dire, comme donner ce que l'on n'a pas, tel est le défi relevé par les personnages du Square.

8. Francis Jacques, Dialogiques II. L'espace logique de l'interlocution, Paris, Presses universitaires de France, coll. "Philosophie d'aujourd'hui », 1985, p. 165.

9. Voir Victor Schmidt, Les dialogues de Platon, Paris, Presses universitaires de France, 1947.

10. Jacques Lacan, Le séminaire VIII. Le transfert, Paris, Seuil, 1991, p. 100. Désormais, les références à cet ouvrage seront indiquées par le sigle $T$, suivi de la page, et placées entre parenthèses dans le corps du texte. 


\section{Le square (1955)}

Pour la réédition du Square en 1989, Marguerite Duras précise la condition des personnages (et non leur caractère, selon la terminologie qu'emploie Diderot à propos du drame bourgeois) et le projet original d'écriture:

C'étaient des bonnes à tout faire, les milliers de Bretonnes qui débarquaient dans les gares de Paris. C'étaient aussi les colporteurs des petits marchés de campagne, les vendeurs de fils et d'aiguilles, et tous les autres. Ceux - des millions - qui n'avaient rien qu'une identité de mort. Le seul souci de ces gens c'était leur survie: ne pas mourir de faim, essayer, chaque soir, de dormir sous un toit. C'était aussi de temps en temps ParLER. Parler du malheur qui leur était commun et de leurs difficultés personnelles. Cela se trouvait arriver dans les squares, l'été, dans les trains, dans ces cafés des places de marché pleins de monde où il $\mathrm{y}$ a toujours de la musique. Sans quoi, disaient ces gens, ils n'auraient pas pu survivre à leur solitude ${ }^{11}$.

Cueillir la chance d'un dialogue en fin d'après-midi dans un square, c'est ce que fait le voyageur de commerce. Pour la jeune fille, il s'agit de travailler à comprendre comment on fait pour (sur)vivre, de développer une stratégie volontariste de promotion sociale, d'accès à l'existence personnelle et au bonheur que lui procureraient l'acquisition d'un fourneau à gaz et la conquête d'un mari. L'affleurement du désir dans sa vie commence par l'espace qu'ouvre la parole: «Parler, par exemple, pour moi est une sorte d'aubaine. [...] Lorsque les gens ont envie de parler cela se voit très fort, et c'est bien le curieux, cela n'est pas bien vu en général. Il n'y a guère que dans les squares que cela semble naturel ${ }^{12}$.» Savoir dire la force de ce désir et la vulnérabilité de qui l'éprouve promet une intelligence du dire qui délie la langue du voyageur de commerce: «Il m'avait semblé que vous étiez une personne plus indiquée qu'une autre pour comprendre ce qu'on veut dire» $(S$, p. 490).

La politesse du langage, l'extrême attention à autrui, la courtoisie scrupuleuse qui excède la simple civilité renvoient à un horizon possible de l'usage de la parole chez les bonnes, à la façon

11. Marguerite Duras, Le square [1955], Paris, Gallimard, coll. «Folio», 1989, p. 5.

12. Marguerite Duras, Le square, Paris, Gallimard, coll. "Quarto», 1997, p. 481. Désormais, les références à cet ouvrage seront indiquées par le sigle $S$, suivi de la page, et placées entre parenthèses dans le corps du texte. 
dont «elle[s] parlerai[en]t si elle[s] pouvai[en]t le faire» et à l'apprentissage de la parole par l'écoute. Le square, Duras l'a écrit après avoir écouté les gens se taire dans les squares, entendu le désir muet de bavarder, désir si fort que «[...] ça peut vous rendre même un peu malade, vous donner comme un peu de fièvre. [...] il semble qu'on pourrait se passer de tout, de manger, de dormir plutôt que de bavarder» $(S$, p. 493$)$. Le bavardage n'a rien de gratuit, de trop léger, il imprime une liberté et une disponibilité de parole, issues du désir, il n'a rien du ragot ni du on-dit à la Pinget. Il laisse aller à loisir la pensée, l'imaginaire, les impressions et les évocations. C'est le débridé qui fait sa richesse, le décousu sa plasticité; l'absence de finalité explicite lui confere un don d'hospitalité à autrui et, au bout du compte, assure un sens, une orientation, hors direction et sans boussole. C'est en pratiquant le culte de l'instant présent que le représentant de commerce se dispose à ce moment d'échange crépusculaire, à cette chance de parler non seulement $\grave{a}$, mais avec autrui. Le dialogue se construit dans l'écoute et la reconnaissance de l'altérité de l'interlocuteur tout comme de cette part de soi-même que l'interlocuteur, par son écoute, révèle: «Sans doute ne peut-on rien vous apprendre de ce que vous ne pouvez voir encore, mais la tentation est grande d'essayer quand même de le faire» $(S$, p. 478). Tentation si grande qu'on y cède : «je ne parle pas seulement de ce que vous savez que vous attendez, mais aussi de ce que vous ne savez pas que vous attendez. De quelque chose de moins immédiat que vous attendez sans le savoir» $(S$, p. 503). L'écoute ne découvre pas seulement les motifs et les mobiles d'un crime: ici, "l'écoute parle», comme disait Barthes, car «[1]'homme parlant [...] ne pré-existe pas au langage et se constitue comme sujet au fur et à mesure qu'il parle, écoute, ou mieux encore parle l'écoute qu'il imagine à sa propre parole ${ }^{13}$ ».

\section{L'amante anglaise (1967)}

Ainsi, dans L'amante anglaise, l'interrogateur assiste Claire: "- Si je savais comment répondre je le ferais. Je n'arrive pas à mettre de l'ordre dans mes idées. - Peut-être y arriverons-nous quand même ${ }^{14}$ ?»; "—C'est moi qui vous apprends maintenant

13. Roland Barthes, L'obvie et l'obtus, Paris, Seuil, coll. «Tel Quel», 1982, p. 226.

14. Marguerite Duras, L'amante anglaise, Paris, Gallimard, 1967, p. 142. Désormais, les références à cet ouvrage seront indiquées par le sigle $A A$, suivi de la page, et placées entre parenthèses dans le corps du texte. 
que vous auriez pu leur dire [je n'aime pas la viande en sauce] ? — Peut-être» (AA, p. 79).

Ainsi l'objurgation finale "Moi à votre place, j'écouterais. Écoutez-moi... je vous en supplie» $(A A$, p. 187) magnifie la disponibilité de l'auditeur qui renvoie au locuteur sa propre parole, la fait résonner à son âme. Il lui faut une "patience religieuse», sans jugement ni présupposé. L'interrogateur de Claire cherche pour elle, "est complètement "en-allé" dans le crime commis par cette femme. [...] Il est désespéré par elle, pour elle — il a la lenteur du désespoir, celle, religieuse d'un croyant. Au Théâtre Gémier, parfois il montait là sur scène et, lentement, il allait derrière elle et posait ses mains sur ses cheveux et il restait là jusqu'à la fin. Et elle, elle parlait sous ses mains, tout à coup heureuse ${ }^{15} »$. Ce bonheur irradie de la confiance de Claire: «Je vous parle à vous parce que vous ne savez rien et que vous désirez vraiment savoir, tandis que mon mari croyait savoir, c'était du temps perdu que de parler avec lui» $(A A$, p. 180). Le dialogue tisse le lien qui délie la parole, fait parler non pas au sens où l'entendent les inquisiteurs de Pinget mais dans l'acception durassienne et barthésienne du terme: "L'interpellation conduit à une interlocution, dans laquelle le silence de l'écouteur sera aussi actif que la parole du locuteur: l'écoute parle, pourrait-on dire ${ }^{16}$.» C'est le passage de l'intelligence que la mise en scène de Claude Régy privilégie:

On est parti dans une représentation complètement abstraite, c'est-à-dire que les personnages étaient assis au centre du public et que quelque chose, quelqu'un posait des questions, invisible, dans le public, et que, tout d'un coup, peut-être pour la première fois, ce quelqu'un ou ce quelque chose ne représentait pas du tout un personnage, ça représentait une force en action, [...] une intelligence qui essaye d'avancer, qui essaie de voir clair et naturellement ${ }^{17}$.

Il faut toutefois admettre que l'intelligence ne se confond pas avec la compréhension, avec la réduction au même, identifié, mais s'ouvre curieusement et généreusement à l'irréductible, accueille l'étrange et l'étranger. Qu'elle ne s'effraie pas de l'approximatif dans la mesure même où elle substitue à la préhension l'approche, travaille à apprivoiser du non-dit, de l'inavoué, de l'insu: «comme

15. Marguerite Duras, Théâtre de l'amante anglaise, Paris, Gallimard, 1991, p. 18.

16. Roland Barthes, L'obvie et l'obtus, ouvr. cité, p. 223.

17. Claude Régy, "Écriture romanesque, écriture dramatique», Cahiers RenaudBarrault, Paris, n 91, 1976, p. 7. 
si », "peut-être», "une sorte de» sont autant de modalisations caractéristiques du discours durassien et mimétiques d'un mouvement de l'esprit, fait d'humilité intellectuelle et de respect devant la fragilité et l'identité de ce qui est à dire. Le récepteur porte autant la responsabilité du discours tenu que le destinateur: tant qu'il n'est pas l'inquisiteur qui arrache l'aveu, «[c]elui qui écoute ne sera pas simplement le maître du pardon, le juge qui condamne ou tient quitte; il sera le maître de la vérité. Sa fonction est herméneutique ${ }^{18} »$.

On a pu rappeler la familiarité du soupçon de Sarraute avec l'inquisition de Pinget, et le passage opéré par l'intelligence dans les œuvres des deux néo-romancières, Marguerite Duras et Nathalie Sarraute. Proche de la seconde, Claude Mauriac a souvent souligné la fécondité de l'exploration tropismique et des espaces intimes qui bruissent entre la strate de la conscience et celle du subconscient, où le corps et le pulsionnel sont engagés. L'entreprise romanesque dialoguée qui aboutit au Dîner en ville et à $L a$ marquise sortit à cinq heures adresse un clin d'œil ironique à Valéry ou Breton: la gratuité arbitraire du romanesque et l'artifice de la finalisation de toute narration ont cessé d'être les risques du Roman pour devenir leur chance. Feu le romanesque, exit le récit: reste le dialogue explicite entrelacé à un dialogue télépathique autour d'une table dressée pour un dîner en ville ou autour d'un carrefour du cœur de Paris (le carrefour de Buci). La fiction se construit de l'enchevêtrement et du tissage de discours verbalisés selon le rituel de la communication de la mondanité et de monologues intérieurs qui, communiquant d'une façon non conventionnelle, finissent par devenir des «dialogues intérieurs».

\section{Le dialogue intérieur (1957-1963)}

Le projet de Claude Mauriac est inconcevable sans la foi en un phénomène psychique, magique, celui de la communication simultanée de conscience à conscience. Pas même entre les mots, mais en deçà des mots, comme "intérieur» le signifie. Le dialogue intérieur ajoute à la sous-conversation sarrautienne la dimension plurielle de l'entretien, de l'échange entre locuteurs muets et paroles tacites. Les paroles dites coexistent avec celles que l'on tait, le prononcé avec l'esquissé. Dans Le dîner en ville comme dans $L a$

18. Michel Foucault, Histoire de la sexualité, ouvr. cité, p. 89. 
marquise, le monologue intérieur est signalé par la typographie qui l'introduit, les points de suspension, auxquels s'opposent les tirets traditionnels de présentation de la réplique dite. Ainsi deux strates de discours se juxtaposent; c'est ce que signifie la glose, implicite dans l'espace de la fiction mais explicite dans la dimension du texte, de Bertrand Carnéjoux sur Eugénie Prieur: «[...] ce n'est pas à ce que Roland lui disait que répond Eugénie, mais à ce qu'elle se disait ${ }^{19}$ ». Pour que surgisse cette remarque, il faut admettre que les commensaux détectent en chacun de leurs voisins les trajets de leurs monologues intimes et des conversations à haute voix, et reconnaissent quel est le véritable destinataire de chaque parole: «soi-même comme un autre ${ }^{20}$ ", ou l'autre «en chair d'encre et en os de papier».

La forme de ces romans du Dialogue intérieur est strictement dialogale, leur matière intégralement dialogique : la communication entre êtres humains en est la "substantifique moelle», fondant un humanisme spécifique à Claude Mauriac: sans religion, il affirme la foi en des ressources psychiques primitives, prérationnelles, qui justifient le montage du Temps immobile autant que le tressage du Dialogue intérieur. Le personnage incarne allégoriquement la polyphonie intime de chaque parlant, parfois cacophonique, et annonce le je éclaté et pulvérisé qui cherche à se rassembler dans Tu ne t'aimes pas. Il ne se construit qu'en dialogue et n'existe que dans la fiction de parler à un alter ego de fiction. S'il est ici question d'un néo-réalisme, c'est en vertu de ce principe: tous les moi d'un je, indénombrables, s'inventent un/des interlocuteur(s), un $t u$, frère imaginaire, complice ou rival, car il n'existe pas de conscience sans dialogue, ni de voix ou d'effet de voix sans oreille ou effet d'écoute.

Le dialogue intérieur officie en questionnant une anthropologie d'un nouveau type, celle d'une vie en dialogue, selon le titre de l'œuvre de Martin Buber ${ }^{21}$, et se fait l'écho d'interrogations épistémologiques et éthiques qui ont marqué les années 1960 et 1970. «Être veut dire être apostrophé», écrit Martin Buber, qui emploie les termes d'anthropologie philosophique et de polyphonie dans un sens comparable à celui que leur assigne Bakhtine.

19. Claude Mauriac, Le dîner en ville [1959], Paris, Gallimard, coll. «Folio», 1985, p. 172-173. Désormais, les références à cet ouvrage seront indiquées par le sigle $D E V$, suivi de la page, et placées entre parenthèses dans le corps du texte.

20. Nous empruntons à Paul Ricœur le titre de son ouvrage de 1990 (Paris, Seuil).

21. Martin Buber, La vie en dialogue, Paris, Aubier-Montaigne, 1959, p. 115. 
Selon Bakhtine, toute manifestation de l'être est appel à la rencontre: "Toute expérience intérieure s'avère être située à la frontière, elle rencontre autrui, et toute son essence réside dans cette rencontre intense. [...] La vie est dialogique de par sa nature. Vivre signifie participer à un dialogue, interroger, écouter, répondre, être en accord ${ }^{22}$.» La proposition «alittéraire» de Claude Mauriac répond à cet humanisme et suggère de changer les règles du jeu:

Car s'il n'est point d'art sans parti pris, il devient à la longue indispensable de changer les règles du jeu. [...] Littérature nouvelle? Roman renouvelé? C'est vite dit. Racine, je m'en avise, a déjà remarqué et utilisé ce que j'avais cru naïvement découvrir en écoutant Eugénie. De très anciens commentateurs d'Andromaque ou de Bajazet en prenaient note déjà avec admiration, signalant cet acquit de la littérature moderne, écrivant en bas de page qu'ici Hermione et là Roxane répondaient moins à leurs confidentes qu'à leurs propres pensées (DEV, p. 173).

Le simultanéisme mauriacien postule une communauté anthropologique et une communion intersubjective qui nous sauvent de la solitude ontologique et nous rédiment de la désespérance de devoir mourir, puisque nous prolongeons les autres qui pensent et parlent à travers nous et que nous nourrirons à notre tour les descendants de cette fraternité innommable et légendaire. La communication entre les âmes constitue l'expérimentation esthétique du Dialogue intérieur et vérifie dans l'œuvre fictionnelle de l'écrivain l'hypothèse du Temps immobile: à quoi rime l'image du flux temporel, quand la permanence et l'identité sont assurées par le retour du même et de l'ancien dans l'actuel et dans l'autre?

Ainsi le roman dialogué renouvelle non seulement l'art romanesque mais aussi la vision de l'homme: il renonce à la version rationaliste et positiviste. Les années 1960 et 1970 portent l'espoir logique et éthique d'un fondement nouveau de l'humain: la relation, en philosophie, implique non plus «je pense, donc je suis ", mais je te parle, donc nous sommes ${ }^{23}$. Les aperçus de la

22. Cité par Tzvetan Todorov, dans Mikhaïl Bakhtine, le principe dialogique, Paris, Seuil, 1981, p. 148.

23. Nous renvoyons sur ce point à la thèse que nous développons dans MarieHélène Boblet, Le roman dialogué après 1950. Poétique de l'hybridité, Paris, Honoré Champion, 2003. 
psychanalyse lacanienne sur le sujet et le «parlêtre» le «délocalisent »: "Je pense où je ne suis pas, donc je suis où je ne pense pas. $[\ldots]$ Je ne suis pas, là où je suis le jouet de ma pensée. [...] Là où je pense, je ne me connais pas, là où je ne suis pas, c'est l'inconscient; là où je suis, il est trop clair que je m'égare ${ }^{24} »$. Le sujet éprouve son assujettissement à l'inscientia: "Inscitia, c'est l'ignorance brute, tandis que inscientia, c'est le non-savoir constitué comme tel, comme vide, comme appel du vide au centre du savoir. ( $T$, p. 186)» Assujettissement finalement révélateur et libérateur.

Cette dialectique de soi et de l'autre est posée dans d'autres termes et sur d'autres présupposés idéologiques dans les analyses marxistes de Bakhtine. La polyphonie de tout discours et la part de l'emprunté — plus ou moins consciemment approprié — dans les clichés de langue comme dans les opinions ont largement détrôné le sujet, moqué sa toute-puissance et sa clairvoyance: "Initialement je ne prends conscience de moi qu'à travers les autres: c'est d'eux que je reçois les mots, les formes, la tonalité qui forment ma première image de moi-même ${ }^{25}$.» Le "travail de l'autre», qu'il s'agisse de l'Autre "extime» lacanien ou des autres bakhtiniens, accomplit l'opération propédeutique à un échafaudage du troisième type. Ainsi le logicien Francis Jacques conçoit la personne comme construction: "La personne est un faciendum. À la limite, elle serait entièrement œuvre. [...] L'ego est à reconquérir sur la relation interlocutive ${ }^{26}$. . Cette tâche incombe à l'individu, le postule pour mieux le dépasser et le sauver de ce pauvre individualisme égotiste auquel la fin du $\mathrm{XX}^{\mathrm{e}}$ siècle a, semble-t-il, succombé. La génération des romanciers qui a retenu notre attention épouse une attitude rigoureuse, inquiète peut-être, à l'égard de ce qu'il convient déjà d'appeler une société de communication. Faudra-t-il se résoudre à conclure, avec Marc Fumaroli, qu'on entre en communication comme on entre en concentration, avec un numéro de matricule?

Communiquer suppose un égalitarisme de droit entre récepteurs et émetteurs contemporains, en nombre illimité. En échange de cette entrée dans le réseau, ces interlocuteurs interchangeables dépouillent toute qualité personnelle, gage et raison d'être de leur liberté: ils renoncent aussi à mettre en

24. Jacques Lacan, Écrits, Paris, Seuil, coll. «Points», 1966, p. 277.

25. Tzvetan Todorov, Mikhaïl Bakhtine, le principe dialogique, ouvr. cité, p. 148.

26. Francis Jacques, Dialogiques II, ouvr. cité, p. 47 et 384. 
œuvre la rhétoricité du langage, son énergie, son ironie, son esprit, sans lesquels la liberté reste sans voix. On entre en communication comme on entre en concentration, avec un numéro matricule ${ }^{27}$.

Ou bien peut-on fonder la relation à autrui sur l'intersubjectivité et poser définitivement l'autre comme une chance plutôt que comme un risque? Si Pinget dénonçait la rouerie de l'interrogatoire inquisitoire et le dévoiement du logos par la rhétorique sophistique, c'était sans doute pour mieux rappeler la puissance menaçante du langage et l'inscription nécessaire d'un contrat éthique dans l'entretien, contrat dont l'interrogateur de L'amante anglaise ou le bavard du Square s'acquittent. Bavarder n'est pas seulement converser avec vivacité, liberté et brio, mais c'est surtout écouter, relancer, accoucher l'autre d'une parole à venir qu'il ne sait pas encore devoir tenir. «Écoutez-moi... je vous en supplie»: le don de silence est plus prometteur et plus productif que la logorrhée des personnages de romans très contemporains qui monopolisent la parole comme on s'acharne à (se) prouver que l'on existe.

À cet égard, les titres de romans des années 1990 et 2000 sont révélateurs d'un engouement pour le discours, le verbal (à ne pas confondre avec la mode du vocal ou de l'oral qui a marqué les romans de l'entre-deux-guerres, de Céline à Queneau en passant par Beckett ou Butor ${ }^{28}$ ). La conversation (Grasset, 1998) et Nous (Pauvert, 2002) de Lorette Nobécourt, La déclaration (Julliard, 1990), La médaille (Seuil, 1993) et La conférence de Cintegabelle (Seuil, 1999) de Lydie Salvayre, les dialogues d'Amélie Nothomb (Hygiène de l'assassin [Albin Michel, 1992] ou le monologue intérieur déguisé de Cosmétique de l'ennemi [Albin Michel, 2001]) sont poursuivis par le démon que dénonçait Sarraute: leurs personnages tiennent à la parole comme à eux-mêmes, et la perdre signifierait se perdre. Selon les termes de Francis Jacques, les personnages-artefacts n'exhibent pas des sujets fascinés par le son de leur propre voix, mais représentent des personnes en gestation. De ce point de vue, la conversation telle qu'elle se déploie dans les romans les plus récents (comme l'indiquent d'ailleurs les titres apparentés à $L a$ déclaration ou à $L a$ conférence, traversés par la même logorrhée) n'apparaît plus comme un art social, commun

27. Marc Fumaroli, Trois institutions littéraires, Paris, Gallimard, coll. «Folio», 1994, p. 208.

28. Voir Jean-Pierre Martin, La bande sonore, Paris, José Corti, 1998. 
aux honnêtes gens du même monde, qui éprouve l'intégration dans un groupe ou une collectivité, pas plus qu'elle n'opère le passage d'une vérité fuyante et labile, fragile et précieuse. Les années antérieures tenaient à autrui, ne pariaient pas sur le moi solipsiste, mais en faisaient un produit dérivé de l'autre. Les romans de la société de communication, eux, sont davantage le lieu où sévit le mot d'ordre de la singularité, le diktat absurde de l'individualité: soyez vous-même, comme si l'on pouvait penser sans matière et s'imposer sans épreuve, celles qu'incarnent l'autre et sa différence.

C'est sans doute dans certaines écritures dramatiques contemporaines que l'on trouve cet intérêt et cette attention pour la parole partagée dont les romans dialogués que nous avons évoqués faisaient preuve. Les pièces de Philippe Minyana, comme Chambres ou Inventaires ${ }^{29}$, ou les travaux d'écriture de François Bon, essais transgénériques eux aussi jusqu'à Daewoo, attestent une inquiétude et une espérance perpétuées à l'égard de la parole tenue et ténue: "La conversation vous met d'emblée dans une perspective ouverte, tout ce qu'on suggère au bout des phrases, et qui devient muet si on se contente de transcrire. C'est cela qu'il faut reconstruire, seul. [...] J'appelle ce livre roman d'en tenter la restitution par l'écriture, en essayant que les mots redisent aussi ces silences ${ }^{30}$.» Le personnage sera élaboré à partir d'une "construction de mots pour mettre en avant, oui, sa façon de dire les mots ${ }^{31} »$. Le souci poétique d'un effet d'authenticité ne peut que se combiner avec le soin accordé à la résonance de la parole au-delà de la phrase...

29. Philippe Minyana, Chambres. Inventaires, Paris, Théâtrales, 1986-1987. On peut remarquer une évolution de la scène; soit elle accueille de plus en plus de monologues qui forment un "dialogue intérieur», donc de discours; soit elle remplace la représentation du drame par sa narration, comme dans le théâtre épique de Daniel Danis, et en particulier $E$, la dernière pièce de lui créée au théâtre de la Colline à l'hiver 2005.

30. François Bon, Daewoo, Paris, Fayard, 2005, p. 48.

31. François Bon, ouvr. cité, p. 103. 